\title{
Short Communication
}

\section{In vivo test of the vertical phase separation hypothesis: the display of major histocompatibility complex (MHC) class I molecules on membranes of B cells from mice fed high-fat diets}

\author{
Saame Raza Shaikh ${ }^{1}$, Sarah Boyle ${ }^{1}$, Jing $\mathrm{Hua}^{2}$, Zhiping $\mathrm{Li}^{2}$ and Michael Edidin ${ }^{1}$ \\ ${ }^{1}$ Department of Biology, Johns Hopkins University, 3400 North Charles Street, Baltimore, MD 21218, USA \\ ${ }^{2}$ Division of Gastroenterology and Hepatology, School of Medicine, Johns Hopkins University, 912 Ross Building, 720 Rutland \\ Avenue, Baltimore, MD 21205, USA
}

(Received 8 April 2008 - Revised 2 June 2008 - Accepted 26 June 2008 - First published online 14 August 2008)

\begin{abstract}
The membrane vertical phase separation hypothesis predicts that a decrease in plasma membrane acyl chain order will increase major histocompatibility complex (MHC) class I surface expression. The hypothesis is based on modification of plasma membrane acyl chain order in cell culture and has not been tested in vivo. In the present study, we isolated splenic B cells from C57/BL6 mice fed either a normal diet or high-fat diets enriched in SFA or MUFA and assayed for changes in plasma membrane acyl chain order and MHC class I surface expression. Plasma membranes of B cells from MUFA-fed mice had significantly decreased acyl chain order and increased headgroup order. The decrease in acyl chain order correlated with a significant increase in the acyl chain unsaturation of B cells from the MUFA-fed mice. MHC class I surface levels on B cells were not affected by the MUFA-rich diet. This study suggests that the membrane vertical phase separation hypothesis may have limited application in a physiologically relevant setting.
\end{abstract}

Vertical phase separation hypothesis: Major histocompatability complex class I: Plasma membrane order: High-fat diet: Curvature stress

The membrane vertical phase separation hypothesis states that major histocompatibility complex (MHC) class I molecules are 'antidromic' proteins ${ }^{(1)}$. These proteins display a negative correlation between membrane surface expression and lipid packing density ${ }^{(2)}$. The hypothesis is based on experimental modification of acyl chain packing using the polymer polyvinylpyrillidone to expand the plasma membrane, allowing incorporation of different lipids to modify acyl chain microviscosity. With this approach, Muller et al. ${ }^{(1)}$ showed that incorporation of egg phosphatidylcholine into the membranes of mouse splenocytes decreased plasma membrane acyl chain order and this increased MHC class I surface expression. They proposed that lipid-induced changes in acyl chain packing modified the vertical displacement of MHC I molecules ${ }^{(1,2)}$.

We and others have shown that the membrane vertical phase separation hypothesis is valid when cells are treated in culture with heteroacid phospholipids in the presence of polyvinylpyrillidone ${ }^{(3,4)}$. However, when we treated cells with fatty acids complexed to BSA, a condition closer to physiological than the polyvinylpyrillidone treatment, we observed a decrease in plasma membrane acyl chain order which correlated with a decrease in MHC I surface expression, contrary to the prediction of the vertical phase separation model and to the results with heteroacid phospholipids ${ }^{(3,4)}$. In the present study, we tested the model in vivo by feeding mice high-fat diets enriched in either SFA or MUFA to modify the acyl chain composition of B cells. SFA are a major component of the high-fat 'Western diet' and contribute to the development of metabolic disorders $^{(5)}$. In contrast, MUFA are a major component of the 'Mediterranean diet' and have some beneficial health effects for metabolic disorders ${ }^{(6)}$.

Here we show that a high-fat diet enriched in MUFA profoundly decreased the acyl chain order of splenic B cells consequent to an increase in acyl chain unsaturation. We also found that high-fat diets enriched in MUFA increased headgroup order, suggesting the formation of a headgroup region with increased curvature. However, we found no change in MHC class I surface levels, although MHC class II surface levels were lowered with a MUFA-rich diet. The present findings establish for the first time that the membrane vertical phase separation hypothesis has limited relevance in vivo.

Abbreviations: DPH, 1,6-diphenyl-1,3,5-hexatriene; HBSS, Hanks Balanced Salt Solution; MHC, major histocompatibility complex; ND, normal diet; TMA-DPH,

1-[4-(trimethylamino)phenyl]-6-phenyl-1,3,5-hexatriene.

* Corresponding author: Dr Saame R. Shaikh, fax +1 410 516-5213, email saameshaikh@gmail.com 


\section{Materials and methods}

\section{Animals and diets}

Male C57/BL6 mice (4-6 weeks old) were purchased from Jackson Laboratories (Bar Harbor, ME, USA). All animal studies fulfilled the guidelines established by the Johns Hopkins University School of Medicine for humane treatment and euthanasia. C57/BL6 mice were fed for 12-14 weeks as described prevoiusly ${ }^{(7)}$. The diets provided 5.0 or $35.9 \%$ fat by weight, respectively, for the normal diet (ND) or high-fat diet (Bioserv, Frenchtown, NJ, USA). The high-fat diets used in the present study were enriched in SFA $(30.6 \%$ coconut oil $+5.3 \%$ maize oil) and MUFA $(35.9 \%$ olive oil). We verified the acyl chain composition of the diets with GC. The ND was enriched in a mixture of palmitic $(15.2 \%)$, stearic $(3.0 \%)$, oleic $(3.0 \%)$ and linolenic acids $(62.0 \%)$ (Bioserv). The SFA-rich diet contained lauric $(31.1 \%)$, myristic $(27.8 \%)$, palmitic $(14.0 \%)$, oleic $(11.6 \%)$ and linolenic $(11.0 \%)$ acids with $<5 \%$ palmitoleic and stearic acids. The MUFA-enriched olive oil diet contained palmitic $(12.5 \%)$, oleic $(71.3 \%)$ and linolenic $(9.9 \%)$ acids with $<5 \%$ myristic, palmitoleic and stearic acids. The diets also contained the following $(\mathrm{g} / 100 \mathrm{~g})$ : casein (20.0), maize starch (15.0), sucrose (50.0), fibre (5.0), AIN Mineral Mix 76A (3.5), AIN Vitamin Mix 76A (1.0), DL-methionine $(0 \cdot 30)$ and choline bitartate (1.0).

\section{Isolation of splenocytes}

C57/BL6 mice were killed either by $\mathrm{CO}_{2}$ asphyxiation or isofluorane followed by cervical dislocation. Spleens were placed into Hanks Balanced Salt Solution (HBSS) supplemented with $10 \%$ fetal bovine serum. Spleens were gently teased apart with a syringe plunger and passed through a $40 \mu \mathrm{m}$ nylon cell strainer (BD Falcon, Bedford, MA, USA) to create a single-cell suspension. Cells were then washed with HBSS. Erythrocytes were lysed in lysis buffer (ACK Lysing Buffer; Quality Biological Inc., Gaithersburg, MD, USA) followed by a wash in HBSS. Splenocytes were counted by the primary author using a haemacytometer followed by a secondary count by the second author using blinded samples. Dead cells taking up trypan blue were excluded from the counts.

\section{Purification of $B$ cells}

$\mathrm{B}_{220^{+}} \mathrm{B}$ cells were purified from the splenocytes of C57/BL6 mice using magnetic cell sorting according to the manufacturer's protocol. The splenocyte fraction was centrifuged at $300 \mathrm{~g}$ for $10 \mathrm{~min}$ at $4^{\circ} \mathrm{C}$. The supernatant was discarded and the cell pellet $\left(\leq 10^{8}\right.$ cells) was resuspended in $900 \mu \mathrm{l}$ MACS buffer (PBS, 0.5\% (v/v) fetal bovine serum, $20 \mathrm{~mm}-$ HEPES, pH 7.2). The cell suspension was then incubated at $4^{\circ} \mathrm{C}$ for $15 \mathrm{~min}$ with $100 \mu \mathrm{l} \mathrm{B} 220^{+}$(CD45) magnetic beads (Miltenyi, Auburn, CA, USA). After washing, centrifugation and filtration, the cell suspension was loaded on to an LS column (Miltenyi). The column was washed three times with MACS buffer, removed from the magnet and cells eluted with $5 \mathrm{ml}$ MACS buffer. After centrifugation, cells were place in RPMI 1640 1X (Mediatech Inc., Herndon, VA, USA) culture medium supplemented with $10 \%$ fetal bovine serum and $5 \%$ glutamine.
Assessment of membrane order with fluorescence anisotropy

B cells $\left(1 \times 10^{6}\right)$ were washed and labelled with $1 \times 10^{-6} \mathrm{M}$ 1,6-diphenyl-1,3,5-hexatriene (DPH) or 1-[4-(trimethylamino) phenyl]-6-phenyl-1,3,5-hexatriene (TMA-DPH) (Invitrogen, Eugene, OR, USA) in HBSS (Mediatech Inc.) for $5 \mathrm{~min}$ at $37^{\circ} \mathrm{C}^{(3)}$. Under these conditions, DPH or TMA-DPH is localized to the plasma membrane ${ }^{(8)}$. Subsequently, cells were washed and resuspended in HBSS and fluorescence anisotropy was measured on a SLM-48000 spectrofluorimeter (SLM Instruments, Urbana, IL, USA). The probes were excited at $355 \mathrm{~nm}$ and emission was recorded at $425 \mathrm{~nm}$. Fluorescence anisotropy, $A$, was determined as: $A=\left(I_{\mathrm{VV}}-G I_{\mathrm{VH}}\right) /\left(I_{\mathrm{VV}}+2\right.$ $\left.G I_{\mathrm{VH}}\right)$ where $I_{\mathrm{VV}}$ and $I_{\mathrm{VH}}$ are the fluorescence intensities measured, respectively, parallel (vertical-vertical) and perpendicular (vertical-horizontal) to the direction of polarization of the emitted light and $G$ is an instrumentation correction factor defined as $G=I_{\mathrm{HV}} / I_{\mathrm{HH}}$.

\section{$G C$}

Total lipid extracts were prepared from the diets or $\mathrm{B} 220^{+} \mathrm{B}$ cells using the Bligh \& Dyer method ${ }^{(9)}$. Fatty acid methyl esters were prepared by transesterification of the lipids in $14 \%$ boron trifluoride in methanol. The fatty acid methyl esters were extracted into hexane and separated by capillary GC (GC-2010; Shimadzu Scientific Instruments, Columbia, $\mathrm{MD}$, USA) with a ZebronZB-WaxPlus column $(30 \mathrm{~m}$, $0.25 \mathrm{~mm}$ internal diameter, $0.25 \mu \mathrm{m} \mathrm{df})$. Samples $(3 \mu \mathrm{l})$ were introduced at an injection temperature of $250^{\circ} \mathrm{C}$. The column was equilibrated at $180^{\circ} \mathrm{C}$ for $4 \mathrm{~min}$, followed by an increase of $3 \% \mathrm{~min}$ to $207^{\circ} \mathrm{C}$, held for $3 \mathrm{~min}, 2^{\circ} / \mathrm{min}$ to $220^{\circ} \mathrm{C}$, held for $5 \mathrm{~min}, 2 \% \mathrm{~min}$ to $240^{\circ} \mathrm{C}$, held for $3 \mathrm{~min}$. Detection was via flame ionization at $250^{\circ} \mathrm{C}$. Peaks were identified by their retention times in comparison with pure fatty acid methyl ester standards (Nu-Chek Prep Inc., Elysian, MN, USA). Peak areas of identified peaks were summed and each peak area is expressed as a percentage of total peak area in that sample.

\section{Flow cytometry}

Surface staining measurements were made on a FACSCalibur flow cytometer (Becton Dickinson, San Jose, CA, USA) equipped with a $488 \mathrm{~nm}$ argon laser and a $647 \mathrm{~nm}$ diode laser $^{(3)}$. Prior to measurements, cells were blocked with FcR blocking reagent (Miltenyi) to eliminate non-specific binding. Cell samples not expected to bind a given primary antibody were used as controls. B cells were stained with anti-mouse $\mathrm{B} 220^{+}$conjugated to FITC, $\mathrm{CD}^{+} \mathrm{T}$ cells were stained with anti-mouse CD8-PE, and dendritic cells were stained with anti-mouse CD11c-PE (BD Pharmingen, San Diego, CA, USA). To measure surface levels of MHC class I and II, $\mathrm{B} 220^{+} \mathrm{B}$ cells were stained with saturating amounts of antiMHC class II-FITC (M5/114.15.2) (Miltenyi), or anti-MHC class I-Cy5 (20.8.4s). The M5/114.15.2 clone recognizes the alloantigen $\mathrm{I}-\mathrm{A}^{\mathrm{b}}$, the only alloantigen expressed by C57/BL6 mice. $20.8 .4 \mathrm{~s}$, which recognizes $\mathrm{H}-2 \mathrm{~K}^{\mathrm{b}}$ molecules, was purified from hybridoma supernatants using Protein-A affinity chromatography and conjugated to Cy5 dye using a standard kit (Amersham Biosciences, Piscataway, NJ, USA). Dead 
cells were excluded based on forward and side scatter profiles and staining with 7-amino-actinomycin D (BD Pharmingen).

\section{Analysis}

Data were acquired for four to six mice per dietary condition. The number of $\mathrm{B}$ cells from a given animal limited the number of assays that we could perform. Hence, there is variation in the number of data points for each assay. Data were plotted using GraphPad Prism 4.0. Flow cytometry data were analysed with Cell Quest. GC data were analysed using Shimadzu GC solution 2.3. Statistical significance was established using a one-way ANOVA followed by a post hoc Dunnett's multiple comparison test. $P<0.05$ was considered significant. Values are means and their standard errors.

\section{Results}

Mice fed high-fat diets have higher body weights than control

At the start of the study, the mice weighed on average about 20-21 g. After 12-14 weeks of feeding, the average weight for ND mice was 25.9 (SE 0.4) g. Mice fed the high-fat diets enriched in SFA or MUFA had a body weight of 30.4 (SE 0.6) and 31.3 (SE 1.3) g, respectively, corresponding to a statistically signficant $15-19 \%$ increase in body weight compared to ND $(P<0.05)$. Relative to the start of the study, the ND mice showed a $24 \%$ weight gain and the high-fat SFAand MUFA-fed mice had a $46-48 \%$ weight gain.

\section{High-fat diets enriched in MUFA increase splenic weight and} total number of splenocytes of mice

Spleen weights of mice fed MUFA (0.15 (SE 0.02) g) were signficantly higher than weights of spleens from ND mice $(0 \cdot 10(\mathrm{SE} 0 \cdot 0) \mathrm{g})(P<0 \cdot 05)$ and the number of splenocytes per spleen was approximately 1.6-fold higher than the control $(P<0 \cdot 05)$. There was no change in the steady-state numbers of splenocytes from mice on the SFA diet compared to splenocytes from ND animals. B220 ${ }^{+}$B cells made up approximately $36-65 \%$ of the total splenocytes. $\mathrm{CD}^{+} \mathrm{T}$ and CD11c dendritic cells were $<10 \%$ of the total splenocytes. High-fat diets enriched in MUFA significantly increased the number of splenic $\mathrm{B}$ cells $\left(72.9(\mathrm{SE} 7.1) \times 10^{6}\right)$ relative to the ND control $\left(47.2(\mathrm{SE} 3.3) \times 10^{6}\right)$ and SFA $(46.3$ (SE $\left.4.5) \times 10^{6}\right)(P<0 \cdot 01)$. High-fat diets had no effect on the number of $\mathrm{CD}^{+} \mathrm{T}$ cells or dendritic cells. The increase in the number of splenocytes was similar to previous studies that showed diets enriched in PUFA increase the total number of B cells and macrophages ${ }^{(10,11)}$. We did not test for changes in macrophage number.

High-fat diets enriched in MUFA decrease plasma membrane acyl chain order due to increased levels of acyl chain unsaturation

We characterized B cell plasma membrane structure by measuring fluorescence anisotropy of DPH and TMA-DPH, which report on phospholipid acyl chain and headgroup order, respectively (Fig. 1). The fluorescence anisotropy of $\mathrm{DPH}$ significantly decreased in the plasma membrane of $\mathrm{B}$ cells isolated from the MUFA-fed mice (Fig. 1(A)) while TMA-DPH anisotropy was higher (Fig. 1(B)). Compared to the ND control, changes in DPH and TMA-DPH anisotropy were approximately $20 \%$, a profound change for this parameter of plasma membrane structure. Neither DPH nor TMA-DPH anisotropy were altered in the plasma membranes of B cells from mice fed the SFA-rich diet. There was a considerable spread in the anisotropy values, which could be in response to both environmental and metabolic differences between animals, even though they are inbred. We have made similar observations on the lateral organization of membrane molecules in studies with $\mathrm{T}$ cells isolated from transgenic mice fed high-fat diets enriched in unsaturated fatty acids (SR Shaikh et al., unpublished results).

The high-fat MUFA-enriched diet modified acyl chain composition of splenic B cells (Table 1). Relative to the ND, mice fed MUFA had approximately $50 \%$ reduction in the total levels of SFA and a approximately $50 \%$ increase in the total levels of MUFA. Specifically, a MUFA-rich diet significantly decreased the amount of $16: 0$ and increased the amount of $14: 0,14: 1,16: 1$ and $18: 1$ levels. In comparison, mice fed the SFA diet did not show any significant change in the total levels of B cell SFA and MUFA compared to ND. The total levels of PUFA remained constant for all of the dietary conditions.

Major histocompatibility complex class I surface expression is not affected by a MUFA-rich diet but major histocompatibility complex class II levels are lowered

There was no measurable change in MHC class I surface levels for any of the dietary conditions using the 20.8.4s antibody (Fig. 1 (C) and (D)). To verify the results, we also tested a second anti-MHC class I antibody (AF6-88.5) and found no change in surface expression between the ND control and the MUFA-rich diets. For comparison, we tested the effects of high-fat diets on MHC class II surface expression. MUFAfed mice had $25 \%$ lower levels of MHC class II than cells from the ND control (Fig. 1 (C) and (D)). No significant effect was observed with the SFA diet.

\section{Discussion}

The membrane vertical phase separation hypothesis predicts that a decrease in acyl chain order will increase MHC class I surface expression due to a change in the vertical displacement of the protein. The hypothesis is based on decreasing acyl chain order when phospholipids are incorporated into cell membranes, non-physiologically, in the presence of polyvinylpyrillidone. Contrary to the prediction, we show in the present study that a decrease in acyl chain order does not increase MHC class I surface expression (Fig. 1). Thus, we conclude that using physiologically relevant methods to decrease acyl chain microviscosity, such as treatment with fatty acid-bovine serum albumin complexes or in vivo feeding with high-fat diets, does not yield an increase in MHC I surface expression.

We speculate that the difference in MHC class I surface expression between the present study and our previous cell culture study using PUFA-bovine serum albumin complexes may lie in the type of fatty acids used to modify membrane 
(A)

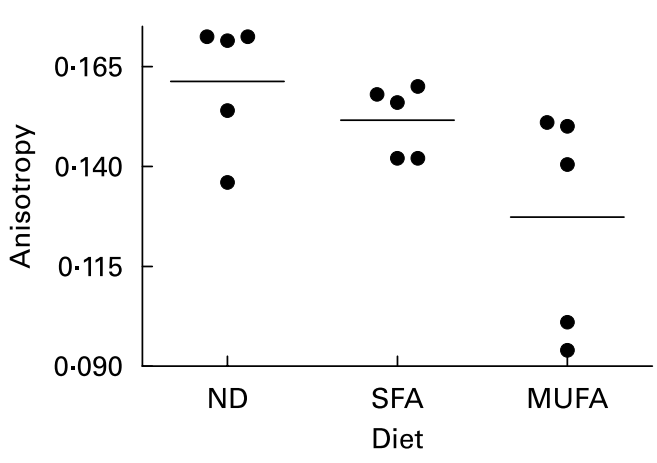

(C)

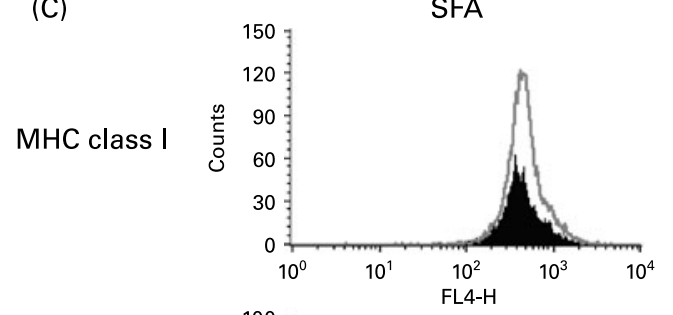

MHC class II

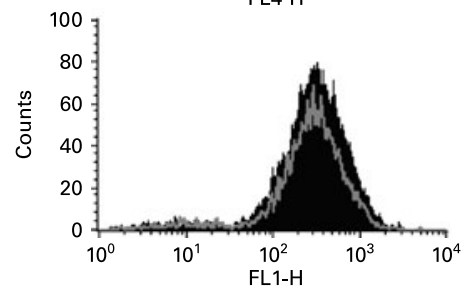

MHC class I

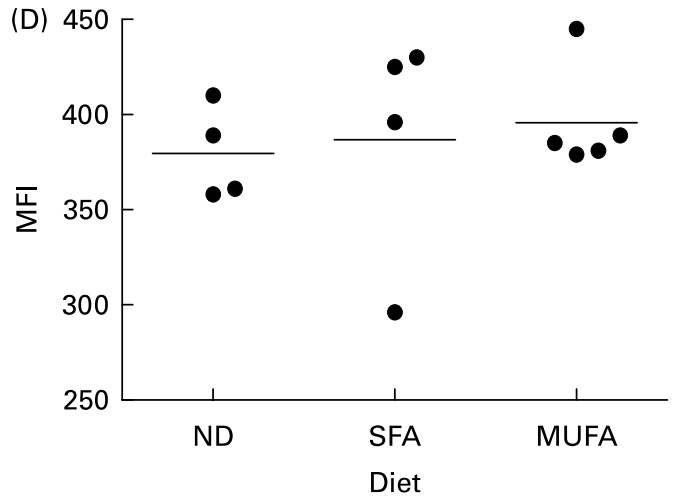

(B)
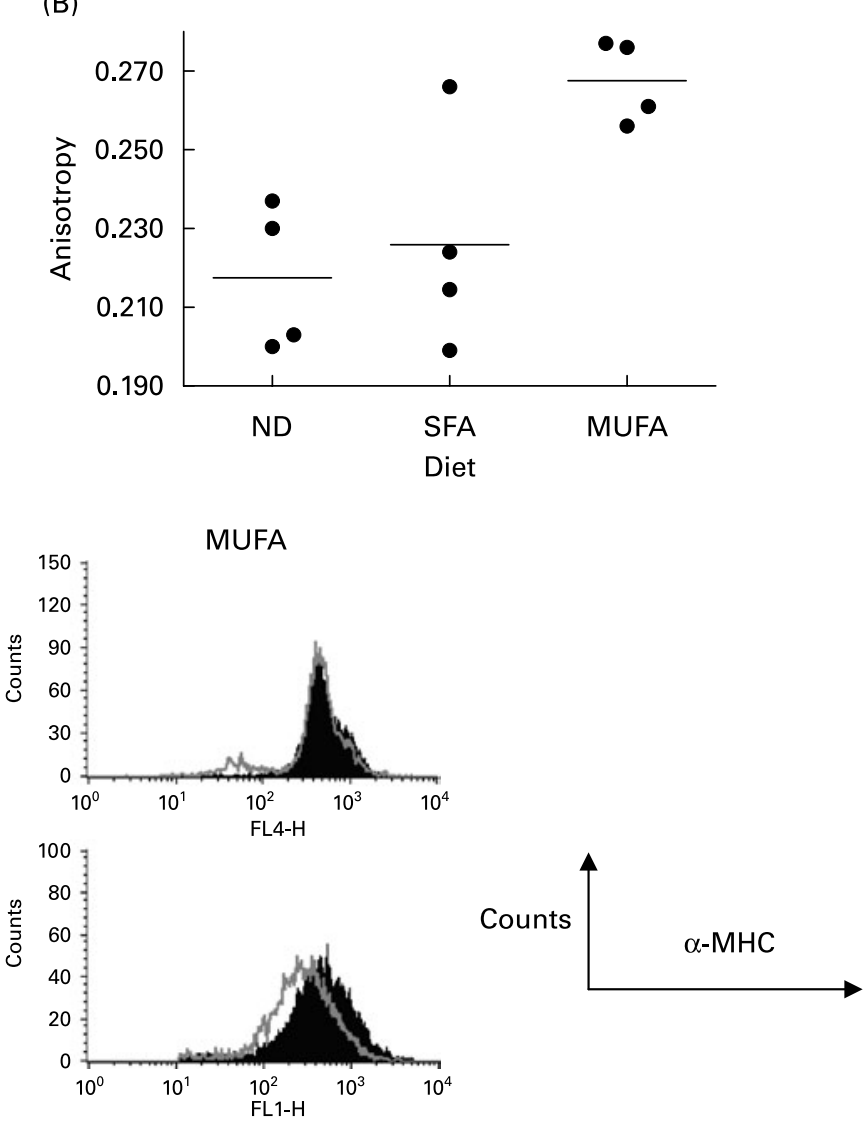

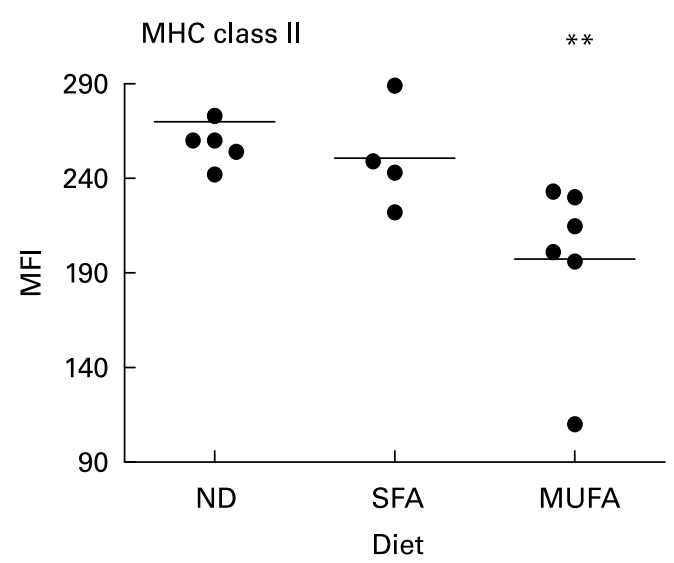

Fig. 1. High-fat diets enriched in MUFA modify B cell plasma membrane properties and protein surface expression. Change in fluorescence anisotropy of (A) 1,6-diphenyl-1,3,5-hexatriene (DPH) or (B) 1-[4-(trimethylamino)phenyl]-6-phenyl-1,3,5-hexatriene (TMA-DPH) incorporated into the plasma membrane of $B$ cells isolated from the spleens of mice fed either a normal diet (ND) or high-fat diets enriched in SFA or MUFA. Fluorescence anisotropy was calculated as described in the Materials and Methods. DPH and TMA-DPH report on acyl chain and headgroup order, respectively. Values for individual mice are plotted with the means depicted by horizontal bars. Mean values were significantly different from those of the ND control group: ${ }^{\star} P<0 \cdot 05$. (C), Sample flow cytometry histograms of fluorescently labelled antibody binding to either major histocompatibility complex (MHC) class I or class II molecules (shaded histograms are the ND control). (D), Fluorescence intensity values (MFI) from flow cytometry histograms for MHC class I and II surface experiments with B cells from differing dietary conditions. Values for individual mice are plotted with the medians depicted by horizontal bars. Median values were significantly different from those of the ND control group: ${ }^{\star \star} P<0.01$.

composition. The present study focused on SFA and MUFA, two fatty acids that are major components of the 'Western' and 'Mediterranean' diets, respectively, that are consumed in abundance. We did not test the effects of PUFA in the present study. In the future, we will test the effects of $n-3$ and $n-6$ PUFA on MHC class I levels in vivo, and its consequences for antigen presentation.
A second finding is that we observe not only a decrease in acyl chain order but an increase in headgroup order of B cells in response to a MUFA-rich diet. This suggests that B cells from MUFA-fed mice have membranes of high curvature stress. High curvature stress can be acquired by a variety of modifications to the membrane, which are not mutually exclusive ${ }^{(12,13)}$. These include changes in the degree of acyl chain 
Table 1. Fatty acid analysis of $B$ cells extracted from spleens of mice (four or five per dietary condition) fed a normal diet (ND) or high-fat diets enriched in SFA or MUFA†

(Mean values with their standard errors)

\begin{tabular}{|c|c|c|c|c|c|c|}
\hline \multirow[b]{2}{*}{ Fatty acid } & \multicolumn{2}{|c|}{ ND } & \multicolumn{2}{|c|}{ SFA } & \multicolumn{2}{|c|}{ MUFA } \\
\hline & Mean & SE & Mean & SE & Mean & SE \\
\hline $12: 0$ & \multicolumn{2}{|c|}{ - } & $4 \cdot 0^{\star *}$ & 0.5 & \multicolumn{2}{|c|}{ - } \\
\hline $14: 0$ & 0.2 & 0.2 & $7 \cdot 6^{*}$ & 1.9 & $4 \cdot 8^{\star}$ & 1.7 \\
\hline $14: 1$ & 0.4 & 0.4 & 0.1 & 0.1 & $4.9^{*}$ & $2 \cdot 1$ \\
\hline $16: 0$ & 37.5 & 5.9 & $29 \cdot 0$ & $2 \cdot 8$ & $16 \cdot 6^{*}$ & 3.9 \\
\hline $16: 1$ & $6 \cdot 7$ & $1 \cdot 8$ & $16 \cdot 1$ & 4.0 & $21 \cdot 0^{*}$ & $6 \cdot 1$ \\
\hline $18: 0$ & $12 \cdot 0$ & $2 \cdot 7$ & $8 \cdot 8$ & $2 \cdot 2$ & 4.7 & 1.7 \\
\hline $18: 1 n-9$ & $6 \cdot 8$ & $4 \cdot 1$ & $4 \cdot 8$ & 1.0 & $25 \cdot 5^{*}$ & $2 \cdot 8$ \\
\hline $18: 2 n-6$ & 3.0 & $1 \cdot 7$ & 1.7 & 0.8 & 3.4 & 0.9 \\
\hline $20: 0$ & 4.8 & $2 \cdot 1$ & $2 \cdot 0$ & 0.9 & $2 \cdot 1$ & 0.7 \\
\hline $20: 1 n-9$ & 4.4 & $1 \cdot 8$ & 1.9 & 0.5 & 1.7 & 0.5 \\
\hline $20: 2 n-6$ & $4 \cdot 1$ & 1.5 & 5.9 & 5.4 & $6 \cdot 4$ & $3 \cdot 7$ \\
\hline $20: 4 n-6$ & 7.9 & 1.4 & 5.4 & $2 \cdot 7$ & $2 \cdot 4$ & 0.4 \\
\hline $20: 5 n-3$ & 0.5 & 0.5 & \multicolumn{2}{|c|}{ - } & \multicolumn{2}{|c|}{ - } \\
\hline $22: 1 n-9$ & $5 \cdot 7$ & 3.5 & $5 \cdot 2$ & 1.0 & $7 \cdot 8$ & 1.8 \\
\hline $24: 0$ & $3 \cdot 1$ & $1 \cdot 7$ & $5 \cdot 6$ & $2 \cdot 8$ & 0.4 & 0.4 \\
\hline $22: 6 n-6$ & 3.5 & 1.2 & 4.0 & $2 \cdot 3$ & $2 \cdot 1$ & 0.8 \\
\hline Total SFA & 57.4 & $5 \cdot 0$ & $51 \cdot 7$ & $7 \cdot 7$ & $28 \cdot 4^{\star *}$ & $6 \cdot 2$ \\
\hline Total MUFA & 24.0 & 4.4 & $32 \cdot 3$ & 1.0 & $53 \cdot 0^{\star *}$ & $7 \cdot 0$ \\
\hline Total PUFA & $18 \cdot 6$ & 1.2 & $16 \cdot 0$ & 8.9 & $18 \cdot 6$ & $3 \cdot 1$ \\
\hline
\end{tabular}

,$-<0.1 \%$.

Mean values were significantly different from those of the ND control group: ${ }^{\star} P<0.05,{ }^{* \star} P<0.01$

†For details of procedures and diets, see Materials and methods. Total cellular lipid was extracted and analysed with GC. Values are expressed as a percentage of total fatty acids.

unsaturation, the length of acyl chains, the position of select double bonds in acyl chains, the degree of headgroup hydration, degree of headgroup repulsion, membrane ionic charge, presence or absence of amphiphilic solutes and levels of select proteins ${ }^{(14)}$. In addition, changes in the levels of sterols, lysophospholipids, phosphatidylethanolamines, glycolipids and diglycerides can also initiate the formation of a membrane of high curvature stress $^{(15)}$. In turn, high curvature stress could imply a propensity for the formation of non-bilayer phases (e.g. hexagonal phase $)^{(16,17)}$. It is important to note that the present data with TMA-DPH (Fig. 1 (B)) only reflects changes in the outer leaflet of the plasma membrane since this probe is not incorporated into the inner leaflet. Therefore, we cannot speculate how a MUFArich diet may modify the inner leaflet.

Finally, we show that a MUFA-rich diet can decrease MHC class II surface expression (Fig. 1 (C) and (D)). The reduction in MHC class II surface expression that we found was similar to that found in cell culture and ex vivo studies with $n-3$ PUFA $^{(18-20)}$. The decrease in MHC class II surface expression could reflect a change in the protein's recycling kinetics, vertical displacement, orientation or conformation of $\mathrm{MHC}$ molecules ${ }^{(21)}$. The vertical displacement model has not been tested in vitro for MHC class II.

In summary, the present study shows that high-fat diets enriched in MUFA, but not SFA, decrease acyl chain order which correlates with an increase in acyl chain unsaturation of splenic B cells. However, the change in membrane order does not correlate with a change in MHC class I surface expression. The present finding is not consistent with the membrane vertical phase separation hypothesis which was based on non-physiological conditions in vitro. The present study points to the challenges of extrapolating findings with non-physiological methods of membrane modulation to in vivo relevance.

\section{Acknowledgements}

We thank Dr Rafat Siddiqui's laboratory (Methodist Research Institute, Indianapolis, IN, USA) for their assistance with GC measurements. The authors contributed to the study as follows: S. R. S. conducted experiments, analysed data and wrote the manuscript; S. B. conducted experiments; J. H. harvested spleens and conducted body weights; $Z$. L. designed the diets and conducted feeding; M. E. designed experiments and wrote the manuscript. This work was supported by grants from the NIH to M. E. (A114584) and to Z. L. (DK068399). There are no potential conflicts of interest.

\section{References}

1. Muller CP, Stephany DA, Shinitzky M \& Wunderlich JR (1983) Changes in cell-surface expression of MHC and thy-1.2 determinants following treatment with lipid modulating agents. J Immunol 131, 1356-1362.

2. Muller CP \& Krueger GR (1986) Modulation of membrane proteins by vertical phase separation and membrane lipid fluidity: basis for a new approach to tumor immunotherapy. Anticancer Res 6, 1181-1193.

3. Shaikh SR \& Edidin M (2007) Immunosuppressive effects of polyunsaturated fatty acids on antigen presentation by human leukocyte antigen class I molecules. J Lipid Res 48, 127-138.

4. Bodnár A, Jenei A, Bene L, Damjanovich S \& Matkó J (1996) Modification of membrane cholesterol level affects expression and clustering of class I HLA molecules at the surface of JY human lymphoblasts. Immunol Lett 54, 221-226.

5. Riccardi G, Giacco R \& Rivellese AA (2004) Dietary fat, insulin sensitivity and the metabolic syndrome. Clin Nutr 23, $447-456$.

6. Lairon D (2007) Intervention studies on Mediterranean diet and cardiovascular risk. Mol Nutr Food Res 51, 1209-1214.

7. Zhiping Li, Soloski MJ \& Diehl AM (2005) Dietary factors alter hepatic innate immune system in mice with nonalcoholic fatty liver disease. Hepatology 42, 880-885.

8. Matko J \& Nagy P (1997) Fluorescent lipid probes 12-AS and TMA-DPH report on selective, purinergically induced fluidity changes in plasma membranes of lymphoid cells. J Photochem Photobiol B 40, 120-125.

9. Bligh EG \& Dyer WJ (1959) A rapid method of total lipid extraction and purification. Can J Biochem Physiol 37, 911-917.

10. Blok WL, de Bruijn MF, Leenen PJ, Eling WM, van Rooijen N, Stanley ER, Buurman WA \& van der Meer JW (1996) Dietary $n$-3 fatty acids increase spleen size and postendotoxin circulating TNF in mice; role of macrophages, macrophage precursors, and colony-stimulating factor-1. J Immunol 157, 5569-5573.

11. Petursdottir DH \& Hardardottir I (2007) Dietary fish oil increases the number of splenic macrophages secreting TNF- $\alpha$ and IL-10 but decreases the secretion of these cytokines by splenic T cells from mice. J Nutr 137, 665-670.

12. Stubbs CD \& Smith AD (1984) The modification of mammalian membrane polyunsaturated fatty acid composition in relation to membrane fluidity and function. Biochim Biophys Acta 779, 89-137. 
13. Jensen MO \& Mouritsen OG (2004) Lipids do influence protein function - the hydrophobic matching hypothesis revisited. Biochim Biophys Acta 1666, 205-226.

14. Mouritsen OG (2005) Life - As a Matter of Fat. The Emerging Science of Lipidomics. Heidelberg: Springer.

15. Cantor RS (1999) Lipid composition and the lateral pressure profile in bilayers. Biophys $J$ 76, 2625-2639.

16. Stubbs CD \& Slater SJ (1996) The effects of non-lamellar forming lipids on membrane protein-lipid interactions. Chem Phys Lipids 81, 185-195.

17. Thurmond RL, Lindblom G \& Brown MF (1990) Influences of membrane curvature in lipid hexagonal phases studied by deuterium NMR spectroscopy. Biochem Biophys Res Commun 173, 1231-1238.
18. Hughes DA, Pinder AC, Piper Z, Johnson IT \& Lund EK (1996) Fish oil supplementation inhibits the expression of major histocompatibility complex class II molecules and adhesion molecules on human monocytes. Am J Clin Nutr 63, 267-272.

19. Hughes DA \& Pinder AC (1997) $n-3$ Polyunsaturated fatty acids modulate the expression of functionally associated molecules on human monocytes and inhibit antigen presentation in vitro. Clin Exp Immunol 110, 516-523.

20. Sanderson P, MacPherson GG, Jenkins CH \& Calder PC (1997) Dietary fish oil diminishes the antigen presentation activity of rat dendritic cells. J Leukoc Biol 62, 771-777.

21. Shaikh SR \& Edidin M (2006) Polyunsaturated fatty acids, membrane organization, $\mathrm{T}$ cells, and antigen presentation. $A m$ J Clin Nutr 84, 1277-1289. 